\title{
Precautions for using quaternary ammonium salt type surfactant on skin
}

\author{
Koji Kubota ${ }^{1}$
}

${ }^{1}$ Affiliation not available

June 19, 2020

\begin{abstract}
Background/purpose: The antiviral activity of quaternary ammonium salt type surfactant has been reported, however, part of those surfactant has enhancement effect of the skin permeability of matters. In this study, the effect of those surfactant on skin barrier function has been evaluated.

Methods: The Transmission Index method, which is a relative value of a substance's skin permeation rate was applied for evaluation of surfactant. The skin injury was evaluated by microscopic observation.

Results: Some cationic and amphoteric quaternary ammonium salt type surfactants significantly enhanced the skin permeability of the substance. However, unlike sodium laurate, there was no skin tissue damage.

Conclusion: The quaternary ammonium salt type surfactant has no skin tissue damage and may be used as a safe disinfectant. However, on the skin which surface-treated by surfactant, the permeation of substances through the skin is increased, which may allow the percutaneous penetration of viruses and other substances. Therefore, be careful when using it.
\end{abstract}

\section{Keywords}

Skin barrier function, Skin permeability, Skin injury, Ionic type of surfactant

\section{Letter to Editor}

The anti-virus activity of surfactants is well known, it is said that attributed to the strong action for the outer coat capsule protein of the virus. (Gerba, 2014),(Perinelli et al., 2019) Recently, a quaternary ammonium salt surfactant was reported to have antiviral activity against SARS-CoV2. (Implementation of Effectiveness Evaluation of Sterilization Methods Against Novel Coronavirus, n.d.) Our study had revealed that quaternary ammonium salt type surfactants have a strong effect on skin barrier function. Therefore, it may require the scrupulous precautions and, in some cases, restrictions when quaternary ammonium salt type surfactants used. In order to inform this fact as soon as possible and to assist in detailed verification by dermatologists and development of formulations for safe use, I report the effect of quaternary ammonium salt type surfactants on the skin barrier function. This manuscript consists of information on changes in the skin permeability of substances due to surfactants, out of the research that attempted a structural chemistry approach to study the mechanism of the effect of surfactants on the skin barrier function. I hope that this report will be useful in countering the threat of COVID-19.

Figure 1 show the TI values of surfactants containing a quaternary ammonium type surfactant. The TI value is a relative value of a substance's skin permeation rate, and when $\mathrm{TI}=1$, it indicates that the permeation rate is the same as that of water-wetted skin. (Okasaka et al., 2018) All experimental results were obtained using a $1 \%(\mathrm{w} / \mathrm{w})$ concentration of surfactant. As detailed in reference article 4, this experiment measures the permeation rate of an indicator substance in surfactant-removed skin after treatment with the surfactant. That is, it should be noted that the skin permeability of the substance is enhanced in the absence of the surfactant. 


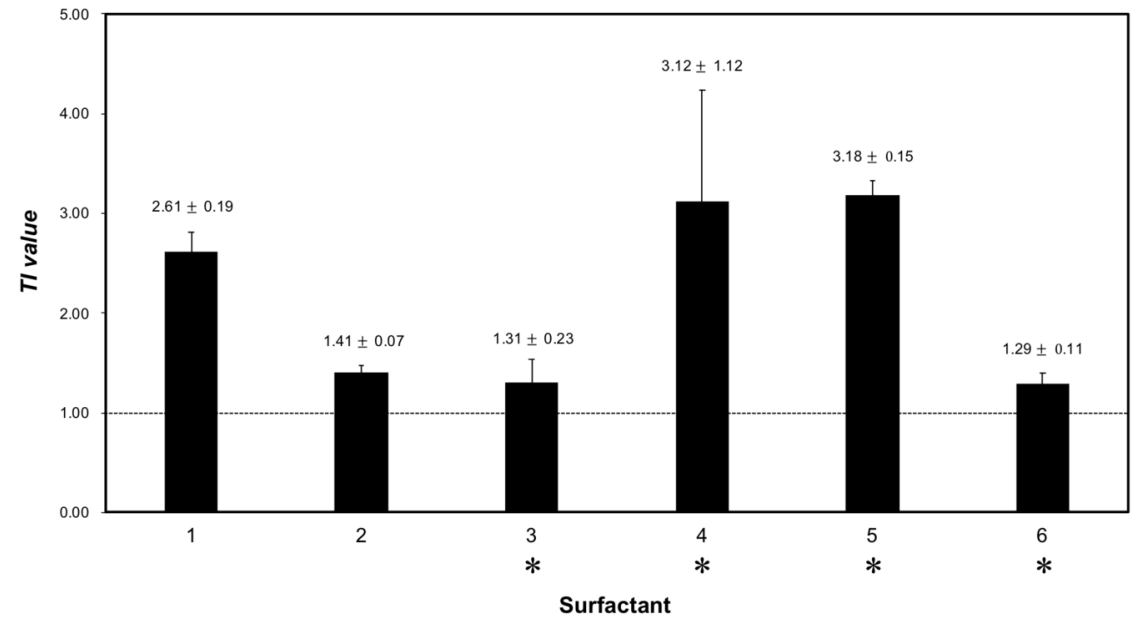

Figure 1: The TI value of surfactant. The TI value on the vertical axis indicates the relative value of the effect on the skin barrier function with water as the standard $(\mathrm{TI}=1)$. Values represent mean and standard error (SE) obtained from 4 or more experiments. 1: Sodium laurate, 2: Sodium lauryl sulphate, 3: Dimethyldistearylammonium chloride, 4: Trimethylstearylammonium chloride, 5: Lauryl Dimethylaminoacetic acid betaine, and 6: Cocamidopropyl betaine. $(*)$ is the quaternary ammonium salt type surfactant.

The asterisk (3-6) in the Figure 1 is quaternary ammonium salt surfactant, 3: Dimethyldistearylammonium chloride, 4: Trimethylstearylammonium chloride, 5: Lauryl Dimethylaminoacetic acid betaine, and 6: Cocamidopropyl betaine. The TI values of 4 and 5 are high, that is, the effect on the skin barrier function is large. 1 is Sodium laurate which is a common soap. With this anionic surfactant, strong tissue damage is observed by microscopic observation. Figure $2 \mathrm{~A}$ shows the microscopic observation image of HE stained skin tissue seriously destructed by 1 . The outermost stratum corneum of the epidermis is lost, and the adhesion between the epidermis layer and the dermis layer is broken. On the other hand, no skin tissue damage is observed with the quaternary ammonium salt surfactant. Figure 2B shows the skin tissue treated by 5 . 2 is Sodium lauryl sulphate, the main component of many neutral detergents. The effect on the skin barrier function is relatively small, but it has injury to the skin tissue. (Kubota et al., 2016)


Figure 2: The microscopic observation image of the HE stained skin tissue. A: the skin tissue treated by Sodium laurate for 1 hour and B: treated by Lauryl Dimethylaminoacetic acid betaine for 1 hour. The thickness of tissue is approximately $20-30 \mu \mathrm{m}$ and objective lens magnification is 20 . The scale bar was added after image acquisition.

Based on the ionic character of the surfactant, 3 and 4 are cationic surfactants and 5 and 6 are amphoteric 
surfactants. Therefore, the mechanisms of antiviral activity and effects on skin barrier function may be fundamentally different between them. Interestingly, the strength of the effect of surfactants on skin barrier function does not depend on the ionic properties of the surfactant. On the other hand, the influence of ionic properties on the antiviral activity is unknown. However, it is important information that some of these are effective in enhancing skin permeation of substances. Since 3 and 6 have a relatively small effect on the skin barrier function, they may be safely applied for sterilization.

The use of surfactants, which strongly influence the skin barrier function, may allow the transdermal entry of an object that is below the cellular level, i.e. viruses. This effect on skin barrier function may be mitigated by mixing with a surfactant having different ionicity, and we are currently studying it. Properly formulated quaternary ammonium type surfactants product may be able to prevent transdermal entry while maintaining anti- SARS-CoV-2 activity.

Because the experiments were performed on mice-extracts skin, please pay attention that these results may differ from human skin.

\section{Acknowledgment}

This work was supported by JSPS KAKENHI Grant Number JP19K14014.

\section{Conflicts of Interest}

The authors report no conflict of interest.

\section{Hosted file}

200618_Main_Document_for_preprint.pdf available at https://authorea.com/users/334183/ articles/460789-precautions-for-using-quaternary-ammonium-salt-type-surfactant-on-skin

\section{References}

Müller, V. (Ed.). (2014). Quaternary Ammonium Biocides: Efficacy in Application. Applied and Environmental Microbiology, 81(2), 464-469. https://doi.org/10.1128/aem.02633-14

Quaternary Ammonium Leucine-Based Surfactants: The Effect of a Benzyl Group on Physicochemical Properties and Antimicrobial Activity. (2019). Pharmaceutics, 11(6), 287. https://doi.org/10.3390/ pharmaceutics11060287

https://www.meti.go.jp/english/press/2020/0415_001.html. https://www.meti.go.jp/english/ press/2020/0415_001.html

Evaluation of anionic surfactants effects on the skin barrier function based on skin permeability. (2018). Pharmaceutical Development and Technology, 24(1), 99-104. https://doi.org/10.1080/10837450.2018. 1425885

The molecular assembly of the ionic liquid/aliphatic carboxylic acid/aliphatic amine as effective and safety transdermal permeation enhancers. (2016). European Journal of Pharmaceutical Sciences, 86, 75-83. https: //doi.org/10.1016/j.ejps.2016.03.002 